\title{
Cohesion Based Personalized Community Recommendation System
}

\author{
Md Mamunur Rashid*, Kazi Wasif Ahmed ${ }^{\dagger}$, Hasan Mahmud ${ }^{\ddagger}$, Md. Kamrul Hasan ${ }^{\S}$ and Husne Ara Rubaiyeat ${ }^{\llbracket}$ \\ Systems and Software Lab (SSL), Department of Computer Science and Engineering (CSE), \\ Islamic University of Technology (IUT), Gazipur, Bangladesh \\ ๑Department of Computer Science, Natural Science Faculty, National University, Bangladesh
}

\begin{abstract}
Our life is totally engaged by the progressive growth of online social networking. Because, millions of users are interconnecting with each other using different social media sites like Facebook, Twitter, LinkedIn, Google+, Pinterest, Instagram etc. Most of the social sites like Facebook, Google+ allow users to join different groups or communities where people can share their common interests and express opinions around a common cause, problem or activity. However, an information overloading issue has disturbed users as thousands of communities or groups are creating each day. To resolve this problem, we have presented a community or group recommendation system centered on cohesion where cohesion represents high degree of connectedness among users in social network. In this paper, we emphasis on suggesting useful communities (or groups in term of Facebook) that users personally attracted in to join; reducing the effort to find useful information based on cohesion. Our projected framework contains of the steps like: extracting sub-network from a social networking site (SNS), computing the impact of amity(both real-life or social and SNS connected), measuring user proclivity factor, calculating threshold from existing communities or groups of a user and lastly recommending community or group based on derived threshold. In result analysis part, we consider the precision-recall values by discarding community or group one at a time from the list of communities or groups of a certain user and checking whether the removed community or group is recommended by our proposed system. We have evaluated our system with 20 users and found $76 \% F_{1}$ accuracy measure.

Keywords-Social network, Community or Group recommendation, Cohesion, Amity factor, User Preferences or proclivity
\end{abstract}

\section{INTRODUCTION}

With the advent of Web 2.0, social networking sites are becoming more widespread and interactive. Face-to-face, voice, email, video communications are traditional medium of interaction between friends, family and relatives [15]. Although, in online social network, two parties initiate communication without any common value between them. They can freely share their personal information with each other without any precondition [13]. In the virtual world, joining or creating communities or groups with common interests and becoming friends are simply clicking of a button [9]. Recommending useful communities or groups to a particular user is a challenge as it is dependent on a lot of factors. If a community or group is worthwhile to a user then the user might be interested to join that community or group. However, profiling user's personal interests is very difficult task as it is influenced by many factors or parameters. We offer a technique of recommending communities or groups based on cohesion which is affiliated by collaborative ranking strategy. It works by determining correlation between communities or groups and user observing user preferences. The overlooked user preferences are inferred from the detected ones. The more cohesive group of community or group has higher linked strength measured in terms of three factors: amity factors, user proclivity rank, community preferences. We have defined these terms in our proposed recommendation system to suggest the user effective communities or groups to join rather than the irrelevant ones. An previous group of study in this field focused on recommending groups or communities or groups on basis of user profile contents or Homophily [21] (user similarity). However, they do not ponder over degree of interaction among users or combinational impact of various factors like user preference and amity impact. The major contributions of the paper are as follows:

(1) We apply user proclivity factor to approximate user's personal interest over groups or communities or groups in social network.

(2) We introduce friendliness or impact of SNS connected and real-life amity to construct users' social associations which greatly affect their choices in social sites. From the research activities [19] concentrating on personalized tweet recommendation, it is vividly noticeable that "including social relationship factors" escalates the correctness of recommendation.

(3) Our model integrates content based statistics as user proclivity choice or factor, social connection and friendliness impact to appropriately ratify user behavior and personality. The investigational result displays refining community or group recommendation performance and the incorporation of all the factors creates an ultimate model dubbed as cohesion impressively outperforming several baseline approaches.

(4) Our suggested recommendation system executes based on automatically derived threshold which is determined based on user profile and distinct factors. As the threshold value is not constant and differs from user to user according to defined factors it can effectively recommend relevant and useful communities or groups to user.

The remainder of this paper is structured as follows. Related work is discussed in Section 2. In section 3, we describe our proposed model for community or group recommendation. Experimental evaluations are presented in Section 4. Finally, we conclude this paper in Section 5. The initial research of this paper was published in the 18th International Conference 
on Computer and Information Technology (ICCIT), Dhaka, Bangladesh, held on 21-23 December, 2015 [1].

\section{RELATED WORKS}

In this chapter, we define the state-of-the-art of social networking terminologies and insights essential to perceive our suggested approach. Social Network Analysis has its origins in both social science and in the broader fields of network analysis and graph theory [21]. From the concept of online social networking, we emphasize on the importance of connectedness (cohesion) among social network users, community or group recommendation systems and their implications.

\section{A. Community Recommendation System}

Community (i.e. Groups in term of Facebook) recommendation is proposing communities or groups for users they might like to join; however, not joined yet. There are some preceding research like anticipating communities or groups for user based on interaction in social networking site. Social graph is engendered on basis of social bonding of user in a social networking site [3]. Several works are motivated by bi-directional communication between users and activeness of friends in the community or group [5]. There are also some effort like recommending friends in social networking sites based on cohesion [2]. Some works endorse groups based on decision trees and feature extraction from user profile [13]. Certain recommendation systems use collaborative filtering methodology for recommending communities or groups [17]. However, current community or group detection techniques either depend on the content study or only cogitate the underlying configuration of the social network graph while detecting communities or groups. As a consequence, these methods fail to find pertinent communities or groups. In a nutshell, there are several limitations in contemporary techniques as follows:

In [3], [5] and [6], they did not ponder over personalization but only the friendliness strength to find communities or groups for a user to connect. User affinity to particular type of community or group was overlooked and the used parameters for calculation were not appropriate enough to validate user behavior. User proclivity rank is the summation of resemblance among list of communities or groups that user has already joined and the groups to join. In our work we consider user's social relation termed as amity factor which is degree of interaction between user and his friends and user proclivity rank. Our experimental results show that it provides better user experience recommending related community or group for individual.

\section{B. Limitation of present Approach}

1) In the first two research works, only the amity factor is used for recommending communities or groups for a user; however, the user proclivity is unnoticed.

2) Collaborative approach for community or group detection is suitable to some extent but doesn't consider the amity strength. Therefore, the collaborative approach lacks intuition in judging rational behavior of user.

3) The constraints used are not suitable enough to ratify users ordinary behavior. In our work, we are considering both amity and users sense of proclivity. We believe that it provides better user experience recommending associated community or group for individual. Business perspective behind our work is to successfully recommend community or group for a user where it is also possible to identify user's taste and to recommend business products for the user.

\section{PROPOSED APPROACH}

In the preceding chapter, we have comprehensively discussed about the prevailing community or group recommendation systems. After evaluating those, we also attempt to prepare a new system for advising communities or groups. In this section, we describe our proposed algorithm based on cohesion.

\section{A. Cohesion in Social Community}

Cohesion is an abstract term that is straightforward to comprehend by intuition but unexpectedly tough to delineate in exact definition. Offhandedly, cohesion is the quantity of all the factors that generates interest in people to join or to be part of a group. [2]. In Social Networking, Cohesion is defined as a connected network and it is considered that network with high degree of connectedness is more cohesive [12]. Cohesion is an essential part of community or group and it is presumed that cohesion would have the same influence on social networking. It is expected that similar individuals interact greatly, at least more often than with divergent people. So, the impact of the cohesion cannot be overlooked in social network and it an effective term to recommend groups or communities or groups for an individual in social networking sites. Our proposed methodology is outlined on the basis of cohesion which is affected by collaborative ranking approach assimilating user's social bonding and activeness in the network. One significant supposition underlying collaborative ranking is that users who accorded in the past are expected to agree again in the future [20]. This assumption allows us to consider past user preferences and forecast communities or groups they might join by checking similar users. In personalized community or group recommendation we can have assumption like below: Assume a user's propensity to join communities or groups depends on user's taste or proclivity and it is impacted by social bonding and correlations. For example, User might be attracted towards particular class of groups or communities providing informative records about different eatery locations and reviews on diverse food items. Therefore, a restaurant related group or community can be a worthy recommendation for that user which is inclined to his own proclivity factor as well as the total number of his/her friends in the same community or group. Amity is an imperative issue here as because user's friends review will be more trustworthy or interesting for him rather than considering review of some unknown individuals. So, user proclivity rank and social connections have great influence on personalized community or group recommendation. In this paper, the cohesiveness or the connectedness among users is recognized on the basis of interaction where we measure amity factor based on carefully chosen parameters. We joined the user proclivity rank with the connectedness among users which attract individual to join a community or group communally termed as cohesion.

\section{B. Framework of proposed approach}

We have outlined a framework of community or group recommendation system centered on cohesion consisting of six functional steps. 


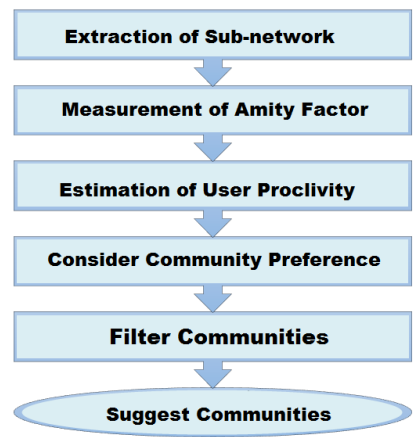

Fig. 1: Outline of Community Recommendation System

\section{Detailed explanation of proposed system}

An essential property of social networks is that people tend to have attributes similar to those of their friends. There are two underlying reasons. Firstly, the process of social influence leads people to embrace behaviors exhibited by those they interact with; this effect at work in many settings where new ideas are diffused by word-of-mouth or imitation through a network of people. A second, distinct reason is that people tend to form relationships with others who are already alike to them [8]. This phenomenon, which is often called selection, has a long history of study in sociology. These factors are utilized by us as because they show a vital role defining amity, bonding or strength, which is obligatory for community or group finding. We are also keeping track of homologous communities or groups as user is already member termed as user proclivity. These factors together provide vivid idea about an individual's mentality and the kind of community or group he/she prefer to join in term of cohesion in this paper.

1) Extraction of sub-network: For the experimental purpose of our proposed system, we take network of a random individual. We collect the available data for our defined factors for a target user.

2) Measurement of amity factor: Amity Factor specifies the numerical quantity of strength of amity between two nodes (friends). In social network, a user has both SNS connected and real-life or social friends. Both types of relationships are significant for assessing amity strength. We compute both SNS connected and social amity factor and they are dubbed as Amity rank or strength Fr. Considering two users $t$ and $t_{1}$, the measurement of amity is dependent on the factors as defined below:

$$
\text { Amity } \operatorname{Fr}\left(t, t_{1}\right)=\frac{\text { SNSAmity }_{\text {factor }}\left(t, t_{1}\right)}{2}+
$$

a) Measuring SNS amity factors: We approximate the connectivity strength among the SNS connected friends on the basis of amity factors as declared previously. To find the degree of strength between two nodes $T$ and $t_{1}$, the factors are defined below:

$$
\operatorname{SNSAmity~}_{\text {factor }}\left(t, t_{1}\right)=\frac{\sum_{i=1}^{n} F_{n}\left(t, t_{1}\right)}{n}
$$

Where, $n=$ number of factors for computing SNS amity strength, To identify the amity factors, a survey [18] was performed which ascertained the parameters having impact on
TABLE I: Parameters to calculate SNS connected amity

\begin{tabular}{|l|l|}
\hline Factors & Formula \\
\hline$F_{1}\left(t, t_{1}\right)$ & Number of mutual friends of $t$ and $t_{1} /$ Total friends of $t$ \\
\hline$F_{2}\left(t, t_{1}\right)$ & $\begin{array}{l}\text { Number of applications used by both } t \text { and } t_{1} / \text { Total number } \\
\text { of applications used by } t\end{array}$ \\
\hline$F_{3}\left(t, t_{1}\right)$ & $\begin{array}{l}\text { Number of photos tagged in common between } t \text { and } t_{1} / \\
\text { Total photos of } t\end{array}$ \\
\hline$F_{4}\left(t, t_{1}\right)$ & Number of post on each others wall / Total post by $t$ \\
\hline$F_{5}\left(t, t_{1}\right)$ & $\begin{array}{l}\text { Number of common events of } t \text { and } t_{1} / \text { Total number of } \\
\text { events by } t\end{array}$ \\
\hline$F_{6}\left(t, t_{1}\right)$ & $\begin{array}{l}\text { Number of messages between } t \text { and } t_{1} / \text { Total number of } \\
\text { messages by } t\end{array}$ \\
\hline$F_{7}\left(t, t_{1}\right)$ & $\begin{array}{l}\text { Number of common likes between } t \text { and } t_{1} / \text { Total number } \\
\text { of likes by } t\end{array}$ \\
\hline$F_{8}\left(t, t_{1}\right)$ & $\begin{array}{l}\text { Number of common communities or groups between } t \text { and } \\
t_{1} / \text { Total number of communities or groups by } t\end{array}$ \\
\hline
\end{tabular}

social relationship. We define eight factors to measure SNS amity strength as shown below on the basis of observation and intuition. Therefore, SNSAmity factor $\left(t, t_{1}\right)$ between users $t$ and $t_{1}$ can be defined as, SNSAmity factor $\left(t, t_{1}\right)=$

$$
\frac{F_{1}+F_{2}+F_{3}+F_{4}+F_{5}+F_{6}+F_{7}+F_{8}}{8}
$$

b) Measuring social amity factors: There exist specific class of friends who do not have much online mutual interactions among them in cyber space via social networking sites. However, they belong to common background like same school, college, or same work place, which can be considered as real-life or social friends. To measure the strength between two users $t$ and $t_{1}$, the factors are defined below:

$$
\operatorname{SocialAmity}_{\text {factor }}\left(t, t_{1}\right)=\frac{\sum_{j=1}^{m} P_{m}\left(t, t_{1}\right)}{m}
$$

Where, $m=$ Number of parameters or factors for calculating social amity; $P_{m}\left(t, t_{1}\right)=$ Parameter value of $m$ for link from $t$ to $t_{1}$ Accordingly,

$P_{1}\left(t, t_{1}\right)=$ Number of common educational institutions for $t$ and $t_{1} /$ Total number educational institutes of $t$.

$P_{2}\left(t, t_{1}\right)=$ Number of common workplaces for $t$ and $t_{1} /$ Total number of workplaces for $t$.

We make an assumption for evaluating social amity. Users who have common background or environment tends to have akin interest or mentality. Those users are termed as real-life or social friends who share the defined common factors and also friends in Facebook. Educational background means from where and what school someone came from. Basically it refers all the schools that someone has been. Generally persons with such common backgrounds can be called as old friends.

$$
\text { SocialAmityfactor }\left(t, t_{1}\right)=\frac{P_{1}\left(t, t_{1}\right)+P_{2}\left(t, t_{1}\right)}{2}
$$

Combining SNSAmity factor $\left(t, t_{1}\right)$ and SocialAmity factor $\left(t, t_{1}\right)$ we can get the amity Fr $\left(t, t_{1}\right)$ which is termed as Amity Factor indicating the connectedness between two friends. Maximum value of amity $\operatorname{Fr}\left(t, t_{1}\right)$ is 1 .

3) Measurement of user proclivity rank: Certain user activities passively redirect users judgments of the usefulness [19]. Assume a user's extra contribution to particular type of communities or groups which reflects their personal judgment of informativeness and efficacy. The user proclivity rank is computed on the basis of user attraction to the certain sort of community or group. All the communities or groups are 


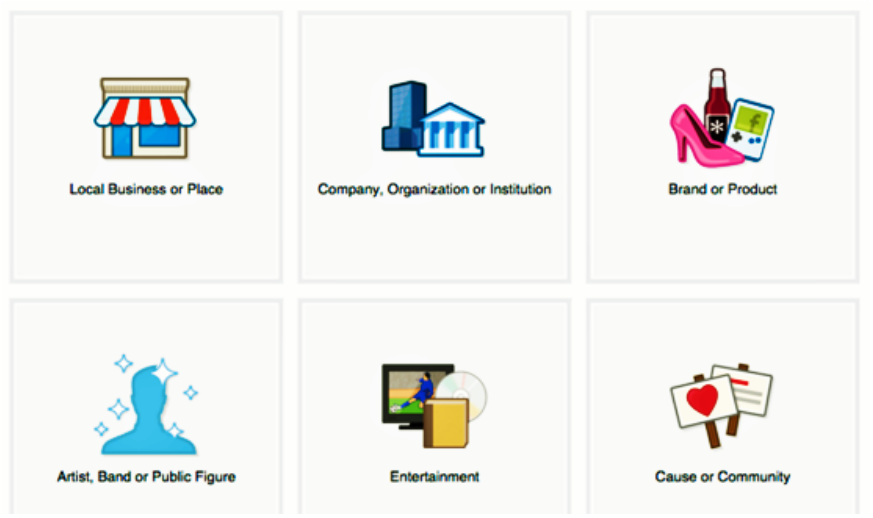

Fig. 2: Types of communities or groups (or groups) in Facebook

TABLE II: Division of user communities or groups into clusters

\begin{tabular}{lcccccc} 
Cluster no. & 1st & 2nd & 3rd & 4th & 5th & 6th \\
\hline Number of Community & 11 & 5 & 9 & 4 & 7 & 2
\end{tabular}

classified into six categories: local business, organization or institution, Brand or product, public figure, entertainment and cause based on intuitive observation in Facebook social network as below figure. The user proclivity rank for a particular community or group is calculated in terms of category of the community or group and number of user community or group present in that category which determines the user preference to that category of community or group. For example: Select a community or group and detect if the community or group belongs to any cluster. User factor of a group for a target user can be defined as: User Proclivity Rank $\left(U_{n}\right)=$

$$
\frac{\text { Number of Communities in the cluster of selected group }}{\text { Summation of communities or groups of target user }}
$$

Suppose, the user belongs to 38 communities or groups which are divided into 6 classes or clusters shown in Table 2. If the community or group to be recommended is $C_{3}$ and it is belonged to cluster no. 3 then

User Proclivity Rank $\left(U_{3}\right)=$

Number of communities or groups in 3rd cluster/cluster no. 3

Total number of communities or groups of target user

$$
=\frac{9}{38}=.237
$$

4) Measurement of community preference: Suppose, we want to recommend communities or groups for a user $t_{x} . t$ is assumed to be the set of users including user $t_{x}$ and his/her friends. Accordingly, $C$ is the list of communities or groups of which the user $t_{x}$ and his/her friends are members. We make an adjacency matrix to represent the belongingness of $t$ to communities or groups $C$ which is defined as $A$. Therefore,

$$
\begin{array}{r}
T=\left\{t_{x} \text { and his / her friends }\right\} \subseteq \text { All groups in social network } \\
C=\left\{\text { Communities where the user } t_{x} \text { and his / her friends belong }\right\} \\
\subseteq \text { All the communities or groups in social network }
\end{array}
$$

Visually we can present this relationship in a bipartite graph $K_{t, C}$. For user $t_{x}$, where $t_{0}$ is the target user having friends

$$
A_{t_{x}}=A_{i, j}=\begin{array}{cccc}
C_{0} & C_{1} & \cdots & C_{j} \\
t_{0} & t_{1} \\
\vdots & t_{i}
\end{array}\left(\begin{array}{cccc}
a_{0,0} & a_{0,1} & \cdots & a_{0, j} \\
a_{1,0} & a_{1,1} & \cdots & a_{1, j} \\
\vdots & \vdots & \ddots & \vdots \\
a_{i, 0} & a_{i, 1} & \cdots & a_{i, j}
\end{array}\right)
$$

Fig. 3: Adjacency matrix of user to community or group relationship

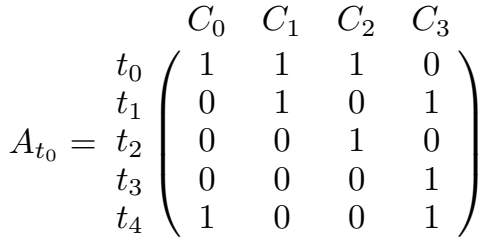

Fig. 4: Finding out possible communities or groups for recommendation

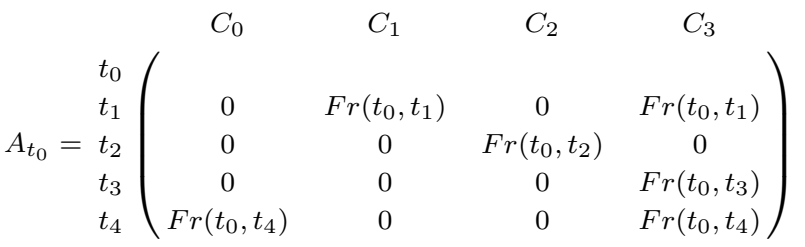

Fig. 5: Calculation of weight factor $\left(C_{3}\right)$

$t_{1} \ldots t_{i}$. So the total no. of friends of $t_{0}$ is $i$. And, $C_{0} \ldots C_{n}$ are collection of communities or groups of which the target user $t_{0}$ and his/her friends $t_{1} \ldots t_{m}$ are members. the corresponding adjacent matrix can be illustrated below: Here,

$$
a_{i, j}= \begin{cases}1, & \text { if } t_{i} \text { if a member of } C_{j} \\ 0, & \text { otherwise }\end{cases}
$$

For a user $t_{x}$, the recommendable set of communities or groups is the set of communities or groups that user $t_{x}$ does not belongs. In effective community or group recommendation system, a subset of these communities or groups should be recommended. Following figure depicts the possible recommendable set community or group is $\left\{C_{3}\right\}$ for user $t_{0}$. Following step is to calculate the community preference $\left(C f_{n}\right)$ of a community or group $n$. Firstly, we determine weight factor $\left(W_{n}\right)$ derived from the amity factors of all the users belonging to a particular community or group $C_{n}$ and multiply it with the User Proclivity Rank $\left(U_{n}\right)$. We replace the elements of $A_{x}$ with corresponding amity factor $\operatorname{Fr}\left(t_{x}, t_{i}\right)$ where $a_{i, j}=1$. As we are recommending for user $t_{x}$, row of that user is omitted from $A_{x}$. Following figure explains the above operation for community or group $C_{3}$.

$$
\text { Weight factor } W_{n}=\sum_{\forall \neq x \in T} F r\left(t_{x}, t_{y}\right) \times A_{y, n}
$$

So the Weight factor of community or group $C_{3}$ could be

$$
W_{3}=F r\left(t_{0}, t_{1}\right)+F r\left(t_{0}, t_{3}\right)+F r\left(t_{0}, t_{4}\right)
$$


Combining the weight factor and user proclivity rank, community preference is calculated for each community or group. The equation for community preference of a particular community or group $C_{n}$ is given below:

Community Preference $(C f n)=$ User Proclivity Rank $\left(U_{n}\right) \times$ Weight factor $\left(W_{n}\right)$

If Community Preference $\left(C f_{n}\right)$ is greater than the Threshold $_{x}$ (calculated for the user $t_{x}$ ) then this community or group is to be processed for further filtering. Threshold can be found by summing up the community preference of present communities or groups of user and then divide it by total number of present communities or groups.

$$
\text { Threshold }_{x}=\frac{\sum_{i=0}^{n} \text { CommunityPreference }\left(C f_{i}\right)}{n}
$$

Here, Community Preference $\left(C f_{i}\right)$ are the Community Preferences of present communities or groups of user $t_{x}$ and $n$ is the total number of communities or groups of user $t_{x}$. As we can see, the value of the threshold varies for user to user is automatically determined.

5) Filter and Suggest Communities: After performing the all steps of our proposed method and filtering based on gender and location, a list of community or group for user is generated. Then finally, we recommend the communities or groups to the user.

\section{Challenges}

There are some challenges we have to face during our research like:

1) Attempting to second-guess a mysterious, perverse and profoundly human form of behavior: the personal response to a work of art is a challenge task for a recommendation system.

2) There are some limitations for determining the factor of amity strength as there are so many things to consider and recommending community or group for a new user will be the most challenging task.

3) The major problem in collecting the Facebook data was privacy concerns. At the same time, the format of the Facebook data was the most congenial to our research method. So, we have collected our data using roster method within a small network of user in Facebook.

4) Our proposed model doesn't solve the fresh start problem. So, user must be member of at least one community or group and user should have some friends because we recommending based on cohesion and user preference.

5) If the target user has no friend or friends do not belong to any community or group then our recommended system fails to calculate the threshold value.

\section{EXPERIMENTAL EVALUATION}

\section{A. Dataset}

There is no API to get user's profile information from Facebook without authorization because of privacy concerns. At the same time, the format of Facebook data is the more congenial to our research. We collected 200 users' data based on their permission and listed the required factors as sample in our database for experimental evaluation. Using the data
TABLE III: Profile Information of user $t$

\begin{tabular}{|l|l|}
\hline Total Friends & 976 \\
\hline Total Apps use & 28 \\
\hline Total photos & 470 \\
\hline Total posts & 212 \\
\hline Total messages & 12203 \\
\hline Total events & 68 \\
\hline Total Likes & 218 \\
\hline Total Communities & 38 \\
\hline Total educational institutions & 3 \\
\hline Total work place & 2 \\
\hline
\end{tabular}

TABLE IV: SNS amity related information of target user $t$

\begin{tabular}{|l|l|l|l|l|}
\hline amity & $t_{1}-t_{2}$ & $t_{1}-t_{3}$ & $t_{1}-t_{3}$ & $t_{1}-t_{4}$ \\
\hline Mutual Friends & 37 & 22 & 48 & 10 \\
\hline Common Apps use & 6 & 10 & 12 & 2 \\
\hline Common photos & 54 & 28 & 66 & 18 \\
\hline Common posts & 31 & 10 & 28 & 8 \\
\hline Common messages & 1030 & 2203 & 1240 & 860 \\
\hline Common events & 8 & 12 & 09 & 3 \\
\hline Common likes & 33 & 28 & 36 & 18 \\
\hline Common communities or groups & 6 & 8 & 6 & 3 \\
\hline
\end{tabular}

TABLE V: Social amity connection informaiton of target user $t$

\begin{tabular}{|l|l|l|l|l|}
\hline Amity connection & $t_{1}-t_{2}$ & $t_{1}-t_{3}$ & $t_{1}-t_{3}$ & $t_{1}-t_{4}$ \\
\hline Common educational institutions & 2 & 1 & 0 & 1 \\
\hline Common work place & 1 & 0 & 1 & 0 \\
\hline
\end{tabular}

TABLE VI: User proclivity information of target user $t$

\begin{tabular}{|l|l|}
\hline Community Type & Number of communities or groups \\
\hline Local business & 11 \\
\hline Organization/Institutions & 5 \\
\hline Brand or product & 9 \\
\hline Entertainment & 4 \\
\hline Public Figure & 7 \\
\hline Cause based & 2 \\
\hline
\end{tabular}

sets we calculate list of possible communities or groups for recommendation using our proposed approach. Below tables display sample data sets for a Facebook target user $t$.

Using the data sets we calculate list of possible communities or groups for recommendation using our proposed approach.

\section{B. Evaluation Metrics}

We corroborate our idea on small network in Facebook. List of present community or group of user is considered. Then a number of communities or groups from the present list of user is eliminated and checked if the community or group is suggested using our offered model. The result is ratified in terms of precision, recall and final score. As we are not using any global threshold value. The value of threshold changes based on users present community or group list according to user proclivity rank and amity factor.

Precision is the ratio of the number of relevant records retrieved to the total number of irrelevant and relevant records retrieved. It is usually expressed as a percentage.

Recall is the ratio of the number of relevant records retrieved to the total number of relevant records in the database. It is generally expressed as a percentage.

$F_{1}$ score(also F-score or F-measure) is a measure of a test's 


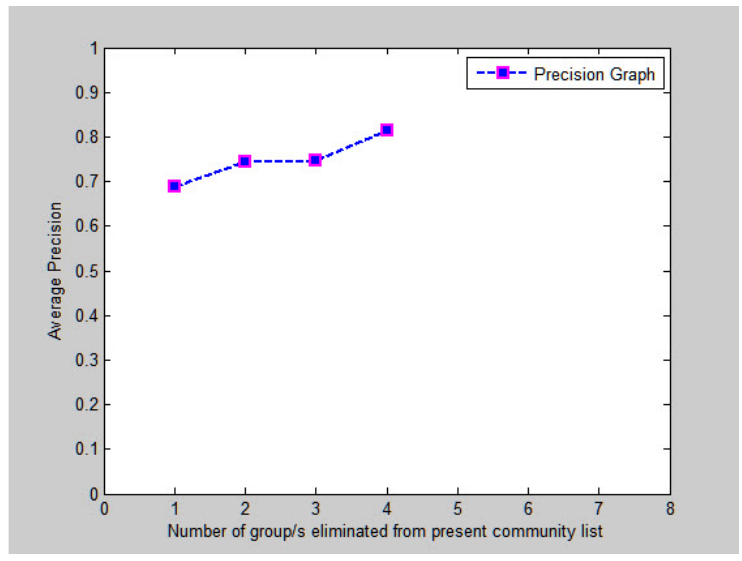

Fig. 6: Average precision graph eliminating half of the communities or groups

accuracy. It considers both the precision and recall of the test to compute the score.

$$
F_{1}=2 \times \frac{\text { precision } \times \text { recall }}{\text { precision }+ \text { recall }}
$$

Firstly, we take one target user and eliminate one random community or group from his/her existing communities or groups and calculate the precision and recall. Then we estimate the average of that and then we eliminate any two communities or groups from his/her community or group list randomly and calculate the mean or average of precision and recall. We do the similar execution until we delete half of the existing communities or groups from the user. Because removing more than half of present community or group from the list increases the possibilities of incorrect suggestion as enough information is already lost. Let us illustrate an example regarding the estimation of precision and recall for better perception. Our target user has 8 communities or groups in his/her present community or group list. We can remove maximum 4 communities or groups from the list to check our recommendation system can identify the relevant communities or groups or not. Following table is displayed as reference. From the table, average precision $=0.7486$ and average recall $=0.7629$. In our case, present communities or groups in the

$$
\text { table[t] }
$$

TABLE VII: Calculation of precision and recall by eliminating user communities or groups

\begin{tabular}{|l|l|l|}
\hline No. of communities or groups eliminated & Precision & Recall \\
\hline 1 & 0.6876 & 0.8750 \\
\hline 2 & 0.7442 & 0.8036 \\
\hline 3 & 0.7473 & 0.7440 \\
\hline 4 & 0.8154 & 0.6289 \\
\hline
\end{tabular}

present community or group list are the relevant (true) records for the user. The newly recommended communities or groups from the universal set which were absent in the present community or group list would be considered as irrelevant record. Suppose, 3 communities or groups are selected from the present community or group list at random and removed. As a consequence, our recommendation system recommended 5 communities or groups in which 3 communities or groups

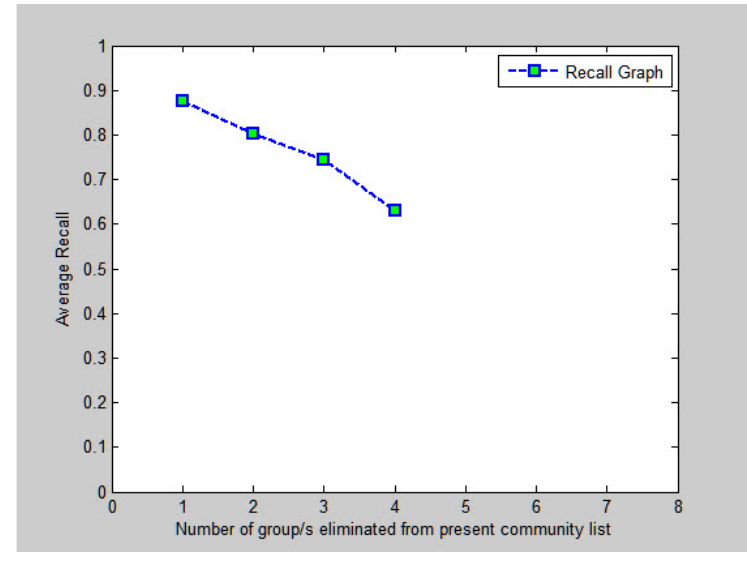

Fig. 7: Average recall graph eliminating half of the communities or groups

are newly recommended (previously not existed in the list) and the last 2 communities or groups are from the removed three (which are considered to be relevant records for user). Here, records retrieved $=$ relevant record retrieved $(\mathrm{A})+$ irrelevant records retrieved $(C)=2+3$

Hence, Precision $=\frac{2}{2+3} \times 100 \%$

On the other hand, RECALL is the ratio of the number of relevant records retrieved to the total number of relevant records in the database. Here, the number of relevant record $=$ relevant record retrieved $(\mathrm{A})+$ relevant records not retrieved (B) $=2+1$

Hence, Recall $=\frac{2}{2+1} \times 100 \%$

Finally, we calculated $F_{1}$ score from the precision and recall value that we got from our experiment to measure the accuracy of our proposed approach.

\section{Method Comparison}

Our works key contribution is to plan and formulate an efficient personalized community or group recommendation system. We compare the proposed model with the prevailing ones intuitively. To the best of our knowledge, we are the first to experiment on real data sets of Facebook users to recommend communities or groups on Facebook using defined parameters. For a target of 20 users, our proposed model successfully recommends correct communities or groups with around $76 \%$ of $F_{1}$ measure accuracy. The average $F_{1}$ score graph for 20 target users is displayed in the following figure. The prevailing approaches do not cogitate the combination of content features which is termed as user preference in our approach and social relationship factors. It is clearly visible from the work of Chen at [19], consideration of social relationship factors increase the accuracy of recommendation to a great extent. Our cohesion based approach considers both the factors of user preference and social relationship providing better recommendation of communities or groups.

The existing approaches using social graph generation in [5] , [6] do not give any idea about accuracy of recommendation. Our proposed approach out performs methods proposed in [15] based feature selection strategy by better 3\% of accuracy. From 


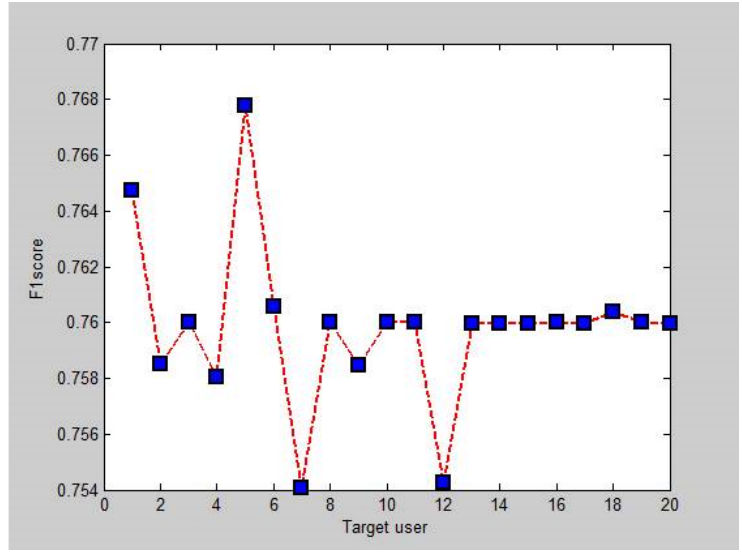

Fig. 8: Average $F_{1}$ score graph for 20 target user

the above results, we conclude that our proposed approach gives better recommendation performance based on cohesion.

\section{CONCLUSION}

Apposite groups or communities are suggested by an efficacious community or group recommendation system to a particular user so that user feels confident enough to join those suggested communities or groups. However, it involves special concern as the psychology of human being fluctuates from person to person in terms of collaboration and interactions. In this paper, we attempt to estimate these bonding and corroboration by defining several parameters to determine most cohesive community or group to be suggested. Our proposed community or group recommendation system is based on user proclivity and also user actives, liveliness or interaction in social networking sites.

This research will assist users to become a member of group or community of their own interest. However, still, there are some boundaries for determining the parameters of amity strength as there are so many things to cogitate and recommending community or group for a new user (a fresh user who has no information in internet). The foremost difficulty while accumulating the Facebook data is privacy concerns. At the same time, the format of the Facebook data is the most congenial to our research necessity. So, we have to collect our data using roster method within a small network of user in Facebook. Our proposed model does not resolve the fresh start problem. So, user must be member of at least one community or group and user should have some friends because we are recommending based on cohesion and user proclivity.

\section{REFERENCES}

[1] K. W. Ahmed, M. M. Rashid, M. K. Hasan, and H. Mahmud, "Cohesion based personalized community recommendation system." IEEE,
2015. [Online]. Available: http://ieeexplore.ieee.org/xpl/articleDetails. jsp?tp=\&arnumber=7488038\&url=http\%3A\%2F\%2Fieeexplore.ieee. org\%2Fxpls\%2Fabs_all.jsp\%3Farnumber\%3D7488038

[2] M. N. Hamid, A. Naser, M. K. Hasan, and H. Mahmud, "A cohesion based friend recommendation system," Springer Journal, Social networking and Mining, Feb. 2014.

[3] S. Kadge and G. Bhatia, "Graph based forecasting for social networking site," International Conference on Communication, Information and Computing Technology (ICCICT), India, Oct. 2012.

[4] B. Krulwich and C. Burkey, "Learning user information interests through the extraction of semantically significant phrases," Mar. 1996.

[5] M. Safaei, M. Sahan, and M. Ilkan, "Social graph generation and forecasting using social network mining." 33rd Annual IEEE International Computer Software and Applications Conference, 2009.

[6] N. Silva, I. R. Tsang, G. Cavalcanti, and I. J. Tsang, "A graph-based friend recommendation system using genetic algorithm," Jul. 2010.

[7] J. Yang, J. McAuley, and J. Leskovec, "Community detection in networks with node attributes," 2013.

[8] D. Crandall, D. Cosley, D. Huttenlocher, and J. Kleinberg, "Feedback effects between similarity and social inuence in online communities," 2008.

[9] Wellman, Boase, and Chen, "The networked nature of community," ITandSociety, 2002.

[10] G. Adomavicius and A. Tuzhilin, "Towards the next generation of recommender systems: A survey of the state-of-the-art and possible extensions," Journal IEEE Transactions on Knowledge and Data Engineering archive, 2005.

[11] C. K. John Breese, David Heckerman, "Empirical analysis of predictive algorithms for collaborative filtering," 1998.

[12] Chin and Chignell, "Automatic detection of cohesive subgroups within social hypertext: A heuristic approach," New Review of Hypermedia and Multimedia, 2008.

[13] J. M. Vitak, "Facebook friends: How online identities impact offline relationships, a thesis submitted to the faculty of the graduate school of arts and sciences of georgetown university in partial fulfillment of the requirements for the degree of master of arts in communication," Culture and Technology, 2008.

[14] P. Melville, R. J. Mooney, and R. Nagarajan, "Content-boosted collaborative filtering for improved recommendations," 2002.

[15] Group Recommendation system for Facebook. On the Move to Meaningful Internet Systems: OTM 2008 Workshops Lecture Notes in Computer Science, 2008.

[16] Gartrell, Xing, and Qin, "Enhancing group recommendation by incorporating social relationship interactions."

[17] W. Y. Chen, D. Zhang, and E. Chang, "Combinational collaborative filtering for personalized community recommendation."

[18] E. carolinllinls, "Success factors of online social networks."

[19] K. Chen, T. Chen, and G. Zheng, "Collaborative personalized tweet recommendation," SIGIR, 2012.

[20] Resnick, Iacovau, and Suchak, "An open architecture for collaborative filtering of newnews."

[21] D. G. Cheliotis, "Social network analysis (sna) including a tutorial on concepts and methods." 